\title{
Violence urbaine, démocratie et changement culturel : l'expérience brésilienne (Partie 2)
}

\section{Angelina Peralva}

\section{(2) OpenEdition}

\section{Journals}

\section{Édition électronique}

URL : http://journals.openedition.org/conflits/706

DOI : $10.4000 /$ conflits.706

ISSN : $1777-5345$

Éditeur :

CCLS - Centre d'études sur les conflits lilberté et sécurité, L'Harmattan

Édition imprimée

Date de publication : 15 octobre 1998

ISSN : 1157-996X

Référence électronique

Angelina Peralva, «Violence urbaine, démocratie et changement culturel : l'expérience brésilienne (Partie 2) », Cultures \& Conflits [En ligne], 29-30 | automne-hiver 1998, mis en ligne le 16 mars 2006, consulté le 30 mars 2021. URL : http://journals.openedition.org/conflits/706 ; DOI : https://doi.org/ 10.4000/conflits.706

Ce document a été généré automatiquement le 30 mars 2021.

Creative Commons License 


\title{
Violence urbaine, démocratie et changement culturel : l'expérience brésilienne (Partie 2)
}

\author{
Angelina Peralva
}

La violence dans la police civile

Les pratiques de torture dans la police civile sont anciennes et assumées par les policiers comme un fait naturel, inhérent à leur métier. Un commissaire interviewé par Benevides (1988: 76) lui explique que la torture est la base même de l'enquête criminelle et qu'elle existe dans tous les pays du monde. " Les policiers ne font que parler des langues différentes (...), ils agissent tous de la même manière. Et s'ils ne le font pas, rien ne se passe. Si le juge était à ma place, il le ferait aussi. Mais là, la justice serait frappée de discrédit. Les juges eux-mêmes n'ont pas intérêt à le faire, ils ne veulent pas venir dans un commissariat pour accrocher ${ }^{1}$ le bandit. Ils veulent faire un procès, ils veulent que vous leur remettiez en mains un dossier tout prêt ". Le commissaire termine en évoquant le sentiment de peur qu'il porte en lui dans l'exercice de son métier. " A qui tous les risques, lors de l'arrestation de l'individu, un risque terrible? Forcer la porte d'une baraque et surprendre le citoyen couché dans son lit, il faut du courage personnel, de la disposition, pour toucher (un très bas salaire) à la fin du mois ! "2

A l'origine, cette violence était limitée, et les débordements punis. En 1962, à Rio de Janeiro, un scandale a éclaté à propos d'une équipe de policiers qui, voulant procéder à un " nettoyage " des clochards, nombreux dans la ville, en ont jeté un certain nombre dans une rivière de la région métropolitaine où ils se sont noyés. Les policiers ont tous été arrêtés, jugés et ont purgé des peines de prison.

Pendant le régime militaire, la violence dans la police civile change d'échelle. C'est à ce moment-là, que se sont formés les " escadrons de la mort ", d'abord à Rio de Janeiro, ensuite à São Paulo. Tolérés, ils ont été néanmoins l'objet de dénonciations systématiques pendant toute la période autoritaire, certains ont été jugés et condamnés. Autant dire qu'ils n'ont pas bénéficié de la même protection que la police 
militaire. Toujours est-il que les escadrons de la mort ont contribué à repousser l'intensité de la violence jusqu'à des seuils auparavant inconnus, qu'ils l'ont naturalisée à travers l'idée d'un apport génétique propre à certains bandits, qu'ils qualifiaient de " bandits nés " et d'irrécupérables ". Dès lors, pour eux, la seule sanction possible était la mort.

Une partie importante des enquêtes menées à São Paulo contre les escadrons de la mort a mis en évidence les liens entretenus entre ces policiers civils et le trafic de stupéfiants. Un journaliste interviewé par Benevides (1983:60), habitué du monde de la police, objecte que la logique mafieuse n'était, cependant, ni exclusive ni dominante au sein de ces groupes. Une seconde orientation, plus idéologique, lui semblait primer dans leur pratique : c'était l'idée de " nettoyage de la société ". Pour la police de São Paulo, Rio de Janeiro avait donné l'exemple, en montrant qu'il ne servait à rien d'arrêter, il fallait tuer. " Je suis allé à Rio voir un commissaire qui est d'ailleurs toujours en service, il s'appelle Rogério Mont Karp, un commissaire célèbre de la Baixada Fluminense. Il m'a expliqué que le système consistait (...) dès l'arrivée [dans une commune de la région], dès le premier jour, quand un nouveau commissaire prenait ses fonctions, à placer deux cadavres à n'importe quel point de la route. Deux bandits, des fois sortis directement de leur cellule (...) ; c'était le premier avertissement, le lendemain encore deux autres, le troisième jour encore deux, alors les bandits disparaissaient. (...) [Ce commissaire] était le propriétaire d'un réseau de stationsservice et il jouait très bien du violon (...) Ceux de São Paulo ont cherché à imiter cette façon d'agir."

Les enquêtes ont permis d'estimer à entre cent cinquante et deux cents le nombre d'exécutions pratiquées à São Paulo contre des prisonniers de droit commun. " 40\% d'entre elles concernaient des gens sortis de la maison d'arrêt Tiradentes, à l'époque. Ils regardaient le dossier d'un prisonnier et ils disaient : " voilà le type avec lequel on va en finir. Monsieur Untel (...) va être probablement libéré grâce à une action en appel menée par l'avocat du Tribunal. "l. Ils prenaient le type, s'arrêtaient dans un endroit isolé quelconque, et l'exécutaient. Nous avons reconstitué des descriptions d'exécutions, où il y avait même une composante étrange, qui était le besoin de montrer qu'ils étaient plus courageux que les bandits. Alors il y a eu des cas où la victime de l'escadron était obligée de se battre physiquement avec le policier. (...) Le type savait qu'il allait mourir. Ils se battaient à mains nues, puis allumaient les phares et le tuaient. Ils manifestaient même du respect pour certaines de leurs victimes, comme dans le cas de ce type qui est mort, une cagoule lui cachant le visage, et qui disait 'vous êtes des fils de putes, des lâches !' [Ils pensaient qu'il] était mort comme un homme, alors ils le respectaient."

La police explique habituellement sa propre violence par la violence de la criminalité. Cet argument amène les policiers à demander de plus en plus de pouvoirs. Ainsi, un commissaire de police d'un quartier de São Paulo, en juin 1982 : " Pour combattre cette criminalité, il faudrait changer la loi à propos de l'abus d'autorité, pour rendre l'action de la police plus facile. Un instrument important, c'est la mise en détention préventive, car si on arrête une dizaine de types au hasard, je vous assure qu'au moins la moitié est déjà inscrite au fichier. " (Fischer, 1985 : 26).

Ceux qui ont connu la police et les délinquants des années soixante pensent que les escadrons de la mort ont joué un rôle décisif dans l'entraînement d'une spirale de la violence. Un journaliste interviewé par Benevides (1983: 64 et s.) évoque un bandit 
d'autrefois, qu'il avait connu à Rio de Janeiro. Il pratiquait des vols à main armée, mais son arme n'était jamais chargée : " Il craignait que le type ne réagisse, et à ce momentlà il serait obligé de le tuer et il serait mis en accusation pour homicide, ce dont il n'avait pas envie. Il savait qu'en volant avec une arme vide, il ne tuerait personne. " Le policier d'autrefois serait aussi moins violent, dit-on. Il rentrait dans le milieu, menait une enquête pendant plusieurs mois, se liait d'amitié avec les proches de l'individu qu'il recherchait, aidait les gens du voisinage, puis à la fin il rédigeait une main courante. Le même journaliste conclut: " Je pense que les escadrons [de la mort] ont stimulé la violence, de même que ce genre d'action policière où le policier, lorsqu'il sort de la voiture, est déjà en train de tirer. Cela a éliminé le bandit qui volait sans armes, le type n'arrive plus à survivre comme un modéré, il faut qu'il soit un radical. " (Benevides, $1983: 69$ ).

Un commissaire interviewé confirme également cette perception: " Le délinquant, le bandit, disons, est devenu violent. Ce que j'appelle la violence, c'est les attaques à main armée, (...) le viol, le meurtre, cette violence est apparue après les escadrons de la mort (...) Le bandit qui purgeait des peines à la maison d'arrêt était un bandit spécialisé, un bandit universitaire. (...) Nous avions à São Paulo des voleurs qui préparaient la clé [dont ils avaient besoin] devant la porte de la maison [qu'ils allaient voler], c'était des champions. On ne pouvait pas s'empêcher d'admirer des types comme ça, c'est un don. Un type qui ne portait pas d'arme, il n'en avait pas besoin, dès que les gens sortaient, il rentrait, il volait tout. Cela existe encore, mais ils sont très rares. " (Benevides : 69). Un autre journaliste interviewé conclut dans la même direction : " Au moment où on quitte ce qu'on appelle le crime intelligent pour passer au crime d'attaque directe, la violence physique vient avec, bien évidemment, car le type réagit, l'autre est obligé de le blesser, de lui faire mal. Il y a eu une massification du crime, du criminel, des délinquants. Les spécialistes (...) étaient des individus, des gens qui avaient leur propre individualité. Aujourd'hui, avec la massification, leur personnalité c'est l'arme, c'est la violence. "

Corruption et inefficacité de la police

Les pratiques de la police, surtout de la police civile, qui accueille quotidiennement tous les types de plaintes de la population, bousculent en permanence les principes de la citoyenneté, en reproduisant et en accroissant les inégalités entre les citoyens. D'un côté, les plaintes des plus démunis ne sont pas prises en considération de manière adéquate et suffisante, de l'autre, ceux qui peuvent payer contournent plus aisément les difficultés et évitent de tomber sous le coup de la loi. (Fischer, 1985 ; Mingardi, 1992). Une telle reproduction des inégalités sociales par le biais du système pénal est ancrée dans des traditions culturelles, certes fondées sur ces mêmes inégalités, mais aussi sur des pratiques qui constituent en elles-mêmes des mécanismes de régulation, qui ont été, dans un passé très proche, propres à des groupes traditionnels. Les commissaires de police ou les inspecteurs entendus par Fischer (1985), qui renvoyaient chez elles des femmes de milieu social modeste venues se plaindre d'avoir été battues par leur mari, et qui leur disaient : " allez y, c'est pas grave, si vous portez plainte, il va se fâcher davantage ", réagissaient en fonction des références propres à ces régulations traditionnelles. De vieux habitants des favelas de Rio, que nous avons nous-même interviewés, racontent que jusqu'à très récemment, des habitants reconnus par les favelados faisaient la police parmi eux. Et c'était bien, disaient-ils, car lorsqu'un mari battait sa femme, elle n'avait pas, comme à présent, à aller au commissariat pour porter plainte. Le problème était tout de suite réglé à l'intérieur du groupe. Aujourd'hui, des commissariats spéciaux d'accueil à la femme remplacent ces régulations 
traditionnelles, non pas nécessairement par la suite d'une modernisation radicale des comportements, mais souvent simplement parce que, par leur existence même, ils favorisent une nouvelle négociation du vieux pacte domestique. (Soares, Soares et Carneiro, 1996). Dès lors, si les progrès de la citoyenneté sont indissociables de la mise en place de structures propres à l'Etat de droit, ils buttent contre des pratiques traditionnelles qui leur résistent.

La reproduction des inégalités sociales par les agents de la police est elle-même ancrée dans les représentations d'une société inégalitaire (Bretas, 1996) où ceux d'en haut ont gardé l'habitude de payer des pourboires aux fonctionnaires de l'Etat juste pour leur rappeler ces différences de fait, que la loi s'abstient d'énoncer formellement. Le problème posé en ces termes, ses conséquences restent néanmoins limitées. Mais la corruption dans la police a, ces dernières années, atteint des niveaux bien plus graves. Elle s'est développée en symbiose avec la criminalité violente et elle la favorise directement. Il est très difficile d'en prendre la mesure exacte, d'autant plus que la justice est elle-même défaillante. Des scandales éclatent régulièrement. Dans les favelas, devenues des repaires de la criminalité et du trafic de drogue, il va de soi que, malgré leurs contradictions apparentes, les policiers frayent avec les bandits. Ces derniers les payent, et à l'occasion les dénoncent. Mais au-delà, les réseaux de la drogue, mobilisent des influences beaucoup plus puissantes.

Encore au-delà, la corruption dans la police est au coeur du trafic illégal d'armes à feu, alors que l'absence de contrôle sur la circulation de ces armes, et l'existence d'armes à la portée de tout le monde, sont indissociables des hauts niveaux atteints à l'heure actuelle par la violence létale. Un certain nombre de faits significatifs illustrent notre propos. L'une des crises les plus récentes dans la police civile à Rio de Janeiro, survenue fin 1994, a été liée au fait que la Division anti-enlèvements - DAS - une division chargée de la lutte contre les enlèvements avec rançon, devenus l'une des formes les plus importantes de la criminalité violente dans la ville (Caldeira, 1996), était soupçonnée d'être elle-même à la tête de ces enlèvements. Lorsqu'il a pris ses fonctions, en 1995, le commissaire Hélio Luz, actuel chef de la police civile de l'Etat de Rio de Janeiro, proche du Parti des Travailleurs, a déclaré : " Désormais, la DAS ne fera plus d'enlèvements. " (Caldeira, 1996b : 61).

Caldeira (1996b : 63-64) indique par ailleurs que quelques grandes opérations de saisie d'armes et de munitions, toutes réalisées en juin 1995 en dehors des favelas de Rio, avaient mis en évidence la participation d'ex-policiers civils ou militaires et d'exmilitaires. De la même manière, l'arrestation de quelques grands trafiquants avait permis de mettre en évidence la participation de policiers (civils, militaires ou de la police fédérale) qui assuraient l'arrivée des drogues, des armes et des munitions dans les centres de distribution au détail, situés notamment dans les favelas.

Violente, et favorisant par conséquent le développement d'une spirale de la violence; corrompue, et favorisant par là les conditions matérielles du développement d'une criminalité violente, la police ne peut être qu'inefficace dans l'exercice de son activité de maintien de l'ordre et de lutte contre la criminalité. Caldeira (1996a) l'a récemment montré dans une étude préliminaire sur les enlèvements contre rançon à Rio de Janeiro. Analysant, à travers un dossier de presse, cent cinquante-sept enlèvements contre rançon survenus dans l'Etat de Rio de Janeiro entre le 1er janvier 1995 et le 31 mai 1996, c'est-à-dire au cours d'une période de dix-sept mois, Caldeira (1996a : 16) a montré que, malgré la sous-estimation probable des données dont il disposait, cette 
branche de l'économie illégale n'a pas mobilisé moins de quatre millions et quatre cent mille réaux (un peu plus de vingt-deux millions de francs). La Division antienlèvements a mené des enquêtes à propos de cent trente-deux enlèvements sur cent cinquante-sept dont la presse a fait état au cours de la période considérée. Pour centdouze cas, le résultat de l'enquête a été négatif; elle a permis d'en élucider vingt. Quarante-cinq personnes ont été arrêtées, dont trente-sept en flagrant délit, mais qui n'avaient toujours pas été mises en accusation. quatre-vingt-dix-neuf suspects se trouvaient en liberté. Seules trois personnes avaient été mises en accusation et se trouvaient sub judice. Caldeira (1996b : 56-57) ajoute que " selon des données de la Centrale d'enquêtes du Parquet, 82 \% des crimes survenus dans l'Etat de Rio de Janeiro, en une période de quatre ans, n'ont pas fait l'objet d'enquêtes de la police civile ".

C'est dans ce contexte de total effondrement de l'appareil policier que l'Opération Rio, par le biais d'une convention signée entre le gouvernement fédéral et celui de l'Etat de Rio de Janeiro, est venue marquer symboliquement la volonté d'une reprise en main de la situation. L'opération s'est déroulée en plusieurs étapes entre novembre 1994 et mai 1995. Son bilan est controversé. Elle a été bien accueillie par l'opinion publique, mais elle a mis en scène diverses tensions entre les logiques de lutte contre la criminalité et l'ordre juridique, comme l'indique Caldeira (1996b). L'armée a été accusée de pratiques de torture à l'encontre de la population pauvre des favelas. Cependant, les cas relevés sont peu nombreux. Il est douteux que ces violences aient atteint des niveaux, même proches, de ceux quotidiennement pratiqués par la police civile et par la police militaire. Les résultats, au niveau de la lutte contre la criminalité, mesurés surtout en termes de saisies de drogues et d'armes effectuées dans les favelas, ont été modestes (Caldeira, 1996b : 69, 70, 71).

Il faut néanmoins observer que l'opération a été déclenchée non pas en fonction d'une volonté de lutte contre la criminalité en général, mais en fonction des niveaux de visibilité du pouvoir des trafiquants des favelas et de l'exacerbation de leur violence à l'encontre de la population en général. Comme le rappelle Caldeira, à plusieurs reprises le couvre-feu avait été imposé non seulement aux favelados, mais assez souvent aussi aux habitants ou aux personnes travaillant dans des quartiers environnants, y compris par la fermeture d'écoles à certaines heures de la journée ou le soir. Dans la déclaration effectuée à ce propos par le président de l'ordre des avocats de Rio de Janeiro, une intervention du gouvernement fédéral se justifiait, dans la mesure où n'étaient plus assurés " les droits de libre circulation, le droit à l'éducation, à la liberté et à la vie " (Caldeira, 1996b : 53).

Du point de vue du rétablissement minimal de tels droits, il n'est pas certain que les résultats aient été complètement négatifs. Nous avons eu l'occasion d'observer l'implantation de l'Opération Rio, dans sa première phase, de l'intérieur d'une favela de la zone sud (la zone des plages et des quartiers riches) de la ville. Même si les habitants ont été en partie réticents à l'égard d'un dispositif qui les discriminait et qui les désignait tout particulièrement, il est certain que l'opération, sans employer des moyens violents, mais par la simple dissuasion, a forcé un recul du commandement du trafic local. Avant même son déclenchement, une masse considérable d'armes ont été déposées dans des voitures aux coffres bien remplis et tout le groupe de tête a quitté les lieux.

L'opération a aussi entraîné une réorientation complète dans les modes de fonctionnement du trafic local. Parmi les trois chefs principaux, deux jeunes frères et 
un proche, l'un a été arrêté et se trouve actuellement détenu dans un quartier de haute sécurité ; l'autre, trop gênant, a été assassiné dans la favela même, et le troisième a déménagé. Le nouveau chef qui leur a succédé, au tempérament plus politique, a établi des rapports d'une autre nature avec la population locale, moins appuyés sur la force et la visibilité des armes et de la violence; plus axés sur la mobilisation d'un sentiment latent d'opposition entre les favelados et les gens " de la rue " ou " du macadam ", manière par laquelle ils désignent la population extérieure à la favela. L'opération n'a pas eu d'effets pratiques du point de vue de la lutte contre la criminalité; elle a contribué à la discrimination de la population des favelas, qu'elle ciblait, (alors que les vrais chefs de ces entreprises criminelles, qui ne vivent pas dans les favelas, n'étaient bien entendu pas visés). Mais il n'est pas certain que ses effets aient été complètement négatifs du point de vue de la vie quotidienne des habitants des favelas.

L'évolution de la criminalité violente

Les taux de criminalité violente ont commencé à s'élever de manière significative au Brésil à partir de la seconde moitié des années soixante-dix. Adorno (1993 : 9) précise la signification de cette notion: " Composent la soi-disant criminalité violente les faits enregistrés concernant des crimes contre le patrimoine (vols et vols suivis d'homicide), contre la vie (homicides), contre la santé publique (trafic et usage de stupéfiants), contre les moeurs (viols), plus les infractions pénales (port illégal d'armes). Sont inclus dans cette catégorie autant les tentatives que les actes accomplis. Le législateur brésilien a établi une distinction entre les crimes et les infractions pénales. Il a classifié comme crime les comportements perçus comme les plus graves, leur réservant des peines d'incarcération allant jusqu'à un maximum de trente ans. Sont considérés comme des infractions pénales des comportements moins graves, qui peuvent faire l'objet d'amendes ou de peines de détention de courte durée. "

Compte tenu du degré de décomposition de l'institution policière et du discrédit dont la police est frappée, il va de soi que les données disponibles sur l'évolution de la criminalité sont fortement sous-estimées. Mais, si on accepte l'hypothèse que ces distorsions restent constantes (Caldeira, 1992), il est possible d'y faire appel pour proposer une première image du problème.

Massena (1986) fait état de l'évolution relative des crimes violents enregistrés dans la région métropolitaine de Rio de Janeiro entre 1977-1980. Entre 1977 et 1978, les taux ont été décroissants dans la région métropolitaine et dans sa périphérie et sont restés stables dans la capitale. Entre 1978 et 1979, ils s'accroissent de façon significative dans la région métropolitaine et dans la capitale, et modérément dans la périphérie. Entre 1979 et 1980, ils décroissent de façon significative dans la région métropolitaine, un peu plus modérément dans la capitale, et très modérément dans la périphérie.

Caldeira (1989, cité par Adorno, 1993 : 4) montre, pour la ville de São Paulo, que la masse des crimes violents par rapport à l'ensemble des crimes enregistrés est passée de $20 \%$ à $30 \%$ entre 1981 et 1987 : " En termes désagrégés, le vol et les atteintes corporelles volontaires sont les modalités de crime qui pèsent le plus sur l'ensemble des crimes violents. A partir de 1983, les vols avec violence vont représenter presque la moitié des faits enregistrés. " Cependant, vaut pour le reste du pays la remarque générale de Caldeira à propos de São Paulo(1992 : 112) - " Ce qui s'est le plus accru à São Paulo", dit-elle, "au cours de la dernière décennie [les années quatre-vingt] et qui préoccupe le plus la population : [c'est] la violence, et non pas seulement le crime " Entre 1979 et 1994, le nombre absolu des homicides au Brésil a triplé : il s'est élevé de 
11194 en 1979 à 32350 en 1994. Le taux pour cent mille habitants est passé, au cours de la même période, de 11,69 à 21,04³. Cette évolution est d'autant plus significative qu'on trouve en général un décalage important entre le taux d'homicide de certaines villes et le taux d'homicide général d'un pays. Aux Etats-Unis, alors que le taux d'homicide s'est maintenu stable au cours des quinze dernières années, plusieurs villes connaissent des taux comparables ou même très supérieurs à ceux de certaines villes brésiliennes. En 1985, par exemple, Washington. D.C. présentait un taux d'homicide de 77,8 pour 100000 habitants, contre 26 à São Paulo ${ }^{4}$. Dans le cas brésilien, on observe au contraire, une cohérence entre l'accroissement du taux d'homicide dans les grandes villes et régions métropolitaines, et son accroissement sur l'ensemble du pays.

Entre 1977 et 1980, le taux d'homicide n'avait pas varié dans l'ensemble de la région métropolitaine de Rio de Janeiro: il s'est modérément accru dans la capitale et de manière un peu plus significative dans la périphérie. En 1980, les homicides constituaient $3,5 \%$ de l'ensemble des crimes violents dans la capitale et $8,3 \%$ dans la périphérie. Une baisse a lieu au tout début de la décennie, puis une courbe ascendante reprend à partir de 1983. Dès lors, dira Coelho (1988), " le mouvement ascendant des taux d'homicide dans la région métropolitaine de Rio de Janeiro est spectaculaire : on passe de 18 faits enregistrés pour 100000 habitants en 1977, à 50 en 1986. Dans la capitale (...) : de 15 faits enregistrés pour 100000 habitants en 1977, à 34 en 1986 ". L'étude, plus récente, de Soares, Sé, Rodrigues et Carneiro (1996), note, après une tendance ininterrompue à l'accroissement des taux d'homicide dans l'Etat de Rio de Janeiro et dans la capitale entre 1985 et 1989, une baisse accentuée pour l'ensemble de l'Etat en 1992. Après avoir atteint un taux de 62,55 homicides pour cent mille habitants en 1989, on régresse à 55,21 en 1992. Dans la capitale, les résultats sont moins significatifs : on enregistre néanmoins une déccélération en 1991 et en 1992. Le taux d'homicide atteint en 1992, dans la ville de Rio de Janeiro, 60,75 pour cent mille habitants, restant stabilisé par rapport à l'année précédente.

Pour le cas de São Paulo, l'étude de Caldeira (1992) montre que l'homicide constitue le type de crime qui a subi le plus fort taux d'accroissement dans les années quatre-vingt, présentant des moyennes annuelles de variation très proches, autant dans la capitale $(13,06 \%)$ que dans les communes de la région métropolitaine (14,76\%). Depuis 1985, ce taux n'a pas cessé de croître et il était en 1989 de 35,58 pour cent mille habitants dans la capitale et 39,28 pour la région métropolitaine. Six ans plus tard, en 1995, le taux d'homicide dans la ville de São Paulo est de 45,59 pour cent mille habitants, restant par conséquent encore très inférieur à celui de la ville de Rio de Janeiro. Caldeira ajoute que déjà au milieu des années quatre-vingt, l'échelle des homicides en faisait la cause de mortalité première chez les adultes. En 1984, les homicides étaient la cause, dans la population âgée de 20 à 49 ans, de $18,5 \%$ des décès contre $8,7 \%$ pour les accidents de voiture.

Coelho (1988) indique, qu'au cours de la période 1981-1986, les taux d'homicide ont été plus élevés dans la ville de São Paulo qu'à la périphérie de sa région métropolitaine. Cette situation s'est modifiée au long de la décennie, comme le montre l'étude de Caldeira (1992) et comme le signale également Pinheiro (1992) : entre mai 1990 et avril 1991, les homicides, selon ce dernier, auraient causé 38 \% des décès dans la capitale, 60 $\%$ dans la périphérie de sa région métropolitaine.

Ces constations induisent une distinction nécessaire concernant les évolutions de la criminalité violente dans son ensemble. Tout d'abord, les crimes violents ne relèvent 
pas toujours de la violence létale. L'étude de Caldeira (1996a) à propos des enlèvements contre rançon dans la ville de Rio de Janeiro montre que sur cent cinquante-sept enlèvements pratiqués entre le 1er janvier 1995 et le 31 mai 1996, seuls quatre ont été suivis de la mort de la victime. En contrepartie, de simples infractions pénales, comme le surf du rail, que nous évoquerons plus loin, peuvent avoir des conséquences létales plus directes et plus importantes.

Mais au-delà, on sait aujourd'hui que le risque d'être volé et celui d'être tué ne sont pas les mêmes pour toutes les catégories de la population. Ce fait simple a été récemment mis en évidence, dans le cas de la ville de São Paulo, par une étude cartographique établie en vue de qualifier les différentes aires de risque dans la ville (Akerman, 1996). Mettant en rapport des données démographiques, socio-économiques, et le nombre de décès causés par homicide, disponibles pour chacun des quatre-vingt-seize districts administratifs de la ville, on constate une opposition tendancielle entre le niveau socioéconomique des districts et les taux d'homicide : Jardim Angela, avec une " note socioéconomique " de 2,02 présente en 1995 un taux d'homicide de 111,52 pour cent mille habitants, supérieur à la ville de Cali, en Colombie. Perdizes, avec la " note socioéconomique " 7 , présente à la même occasion un taux d'homicide pour cent mille habitants de 2,65. Inversement, ce district présente le plus fort taux de vols de véhicules, avec ou sans violences.

$<$ !--SPIP-->

D'autres données montrent une évolution certaine au niveau des formes du crime, notamment le passage, déjà évoqué, du crime d'auteur au crime massifié, où la transgression cesse d'être exceptionnelle pour devenir un problème social ${ }^{5}$; elles indiquent aussi, à l'intérieur de cette massification, l'émergence du crime organisé, soulignée par de nombreux auteurs ${ }^{6}$; et, finalement, elles marquent la dissociation relative, entre ces nouvelles formes de criminalité et les causes des homicides qui, ayant subi, comme nous l'avons $\mathrm{vu}$, une évolution spectaculaire, constituent aujourd'hui la plus grave source d'inquiétude pour la population. En 1996, selon les résultats d'un sondage Datafolha, 63 \% des habitants de la ville de São Paulo craignaient d'être assassinés, 61 \% d'être touchés par une " balle perdue "7

Or, en ce qui concerne les causes de cette criminalité létale, une étude de cas développée dans une des aires les plus criminogènes de la ville de São Paulo, la zone sud, a montré que $48,3 \%$ des auteurs d'homicide, dans cet univers, n'étaient pas des professionnels du crime, mais des personnes qui tuaient dans des situations de tension et de violence interpersonnelle, parce qu'elles disposaient d'une arme à feu à portée de la main. Seuls $24,1 \%$ des cas considérés relevaient du crime professionnalisé stricto sensu. Le rapport entre les décès et l'usage ou le trafic de drogues a été aussi relativisé : $70,1 \%$ des victimes n'avaient pas de casier judiciaire et n'étaient pas usagers de drogues. Les homicides en rapport avec le trafic de drogues occupent le troisième rang parmi les causes les plus courantes de décès dans la zone sud de São Paulo8.

Le profil des victimes d'homicide, cohérent avec celui des auteurs d'homicides, enfin, coïncide dans toutes les études : il s'agit généralement d'un homme jeune ${ }^{9}$. Pour Rio de Janeiro, Cesar Caldeira fait état de chiffres très proches: " l'écrasante majorité des victimes d'homicide (90\%) appartient au sexe masculin. Et le profil de la victime d'un homicide est celui d'un homme pauvre, noir ou métis, entre 18 et 29 ans (avec une concentration dans la plage située entre 18 et 24 ans), décédé dans un lieu public "10. Ces résultats coïncident également avec ceux de Caco Barcellos, concernant les victimes 
de la violence policière ${ }^{11}$. L'homicide touche néanmoins aussi, de manière très significative, les adolescents. Entre 1979 et 1994, 45469 adolescents ont été assassinés au Brésil ${ }^{12}$.

La justice illégale

La généralisation des comportements violents et de la violence dans les relations interpersonnelles est tout d'abord une conséquence naturelle de l'effondrement de l'Etat, incapable de la limiter et de la réguler. Dès lors, on constate de sa part de véritables actes d'incitation à l'autodéfense et des appels à la population à s'armer. Maria Victoria Benevides montre, au début des années quatre-vingt, tandis que le régime militaire était toujours en place, mais en cours d'affaiblissement, l'émergence dans l'espace public de débats fortement médiatisés sur le thème de la violence urbaine et, simultanément, sur le thème de l'autodéfense ${ }^{13}$. " L'auto-armement de la population a été proposé et stimulé par le Secrétaire à la sécurité de [l'Etat de] São Paulo, et justifié, entre autres, par l'évêque de Porto Alegre, D. Vicente Scherer, pour qui 'tous doivent porter des armes, car personne ne doit être idiot et se laisser attaquer' "14. Le Secrétaire à la sécurité de l'Etat de São Paulo a déclaré en toutes lettres : " Tout chef de famille doit avoir une arme chez lui "15.

En même temps que l'incitation à l'autodéfense et au port d'armes, émergent des discours de justification et de légitimation des pratiques violentes, qui abolissent les barrières symboliques empêchant la généralisation de telles pratiques. Maria Victoria Benevides cite les déclaration d'un responsable de la police des polices, Antonio Lucena, à propos des tortures à la suite desquelles un ouvrier de Belo Horizonte avait subi une incapacité définitive de travail ${ }^{16}$. L'ouvrier était accusé de sévices contre un jeune. Le responsable de la police des polices, appelé à se prononcer sur les faits, a déclaré : " Je ne suis pas favorable à la violence policière. Mais je tiens à souligner que, dans ce cas-là, cela s'explique. Si j'étais un juré, et que le père du jeune homme victime des sévices de Jorge [l'ouvrier torturé] l'avait tué et charcuté, je l'aurais acquitté. Plus encore: je décorerais le père "17. $\mathrm{M}$. V. Benevides cite encore le cas de membres du Conseil municipal de la ville de Macaé qui, lors d'un discours devant le Conseil, ont apporté leur soutien aux lyncheurs d'un obsédé sexuel.

La légitimation des pratiques violentes, la tolérance face à la violence marquent toute cette période d'un aveu d'impuissance de l'Etat. Elles ont une portée très large et très générale. Il ne s'agit pas d'une violence de classe, ou de la seule violence des élites, mais de l'acceptation d'une violence que la société n'arrive plus à maîtriser. Un militaire, le colonel Carlos Aloisio Weber, président du Réseau ferré de São Paulo (REFESA), déclare " comprendre parfaitement " le déchaînement de violence des usagers du réseau, qui ont détruit des trains, toujours en retard et en très mauvais état de conservation : 'Si j'étais là, moi aussi je casserais tout... je respecte beaucoup la sainte rage de l'usager, en ce qui concerne les causes, dont la responsabilité nous appartient. " (Benevides, 1983 : 38) Ce type de déclaration, où on n'hésite pas à reconnaître la légitimité des doléances populaires, ainsi que l'impuissance des responsables à y porter remède, est affinée avec le ton du dernier gouvernement militaire, celui du Général Figueiredo, lequel pour sa part déclarait: " Si j'avais à vivre avec un salaire minimum, je me tirerais une balle dans la tête. "

Le discours des responsables institutionnels à l'égard de la violence rencontre un écho favorable au niveau de la société civile. Benevides cite les déclarations du directeur de l'Association commerciale de Santo Amaro (São Paulo): " 'Tout arbre donnant de 
mauvais fruits doit être coupé'. Avec cette citation évangélique, Manoel Rocha Alves (...) dit que beaucoup de ses collègues de bureau soutiennent et défendent les exécutions sommaires comme une forme de lutte contre la criminalité. 'Nous ne supportons plus l'action des marginaux et je crois qu'il est nécessaire que l'escadron [de la mort] fasse quelque chose.' (Folha de São Paulo, 1.5.1983). " Divers sondages ont fait état d'opinions favorables à l'emploi de telles méthodes. Ainsi, celui réalisé à Rio de Janeiro, en 1980, relève $44 \%$ d'opinions favorables aux pratiques de lynchage, car 'si la justice n'agit pas, il faut que le peuple le fasse'. " (Benevides, 1983: 98) Celui réalisé à Recife, en 1986, signale jusqu'à 58 \% d'opinions favorables aux lynchages (Paixão, 1988 : 192).

Dans l'ensemble, le retour progressif à la normalité institutionnelle, de même que la refonte démocratique de la légalité à travers la Constitution de 1988, ont eu comme pendant un fort décalage entre cette légalité retrouvée et son illégitimité de fait. Ce que la légalité démocratique affirmait dans la forme, la large légitimation, dont la violence illégale était l'objet, le niait dans les faits. Sous ce couvert, les pratiques de justice illégale se développent sans retenue tout au long des années quatre-vingt, un phénomène qui se poursuit jusqu'à l'heure actuelle, sous trois formes principales. Premièrement, les lynchages constituent ou bien une modalité spontanée de réaction collective à ce qu'un groupe occasionnel ressent comme une menace ponctuelle contre sa propre sécurité ; ou bien, de manière plus complexe, l'acte de vengeance perpétré par une communauté plus stable, un groupe de voisinage par exemple, face à l'agression subie par l'un de ses membres. Deuxièmement, les chacinas sont des meurtres perpétrés, non pas contre un individu isolé, mais contre tout un groupe de personnes, pour des raisons souvent mal élucidées. C'est devenu depuis quelques années une forme de violence très importante dans les quartiers pauvres et les zones périphériques de São Paulo. Troisièmement, des formes variées de vigilantisme peuvent prendre la forme de pratiques illégales dans certaines entreprises de sécurité privée, qui en appellent à la manière forte ; ou bien il s'agit de groupes qui font la police, avec des méthodes extrêmement violentes, généralement au sein des quartiers pauvres, ou près des espaces commerciaux, à la demande des commerçants. Ils sont connus à São Paulo comme des " justiciers ", à Rio de Janeiro comme des " exterminateurs ".

Nous ne traiterons néanmoins que de deux de ces formes - les lynchages et le vigilantisme, aucune enquête sur les chacinas n'étant pour l'instant disponible, à notre connaissance. Nous nous limiterons à rappeler, sur ce point, les événements d'août 1993, au cours desquels vingt et un habitants de la favela Vigario Geral , à Rio de Janeiro , ont été assassinés par un groupe paramilitaire formé par des policiers militaires et par des ex policiers militaires. Ces derniers vengeaient ainsi, sur la personne des favelados, choisis de manière aléatoire, la mort de deux agents de police, décédés quelques jours auparavant lors d'un affrontement avec des trafiquants de drogue. Ce cas a acquis une notoriété particulière, car au moment de l'enterrement des deux policiers, leurs collègues ont levé collectivement le bras pour leur jurer vengeance, et cela devant les caméras de télévision. D'autres cas, avec un nombre plus réduit de victimes, surviennent régulièrement, en particulier pendant les week-ends, dans les quartiers pauvres de la périphérie de São Paulo. La presse se limite à en dresser une liste monotone, en indiquant le quantième de l'année, alors que pour sa part, la police déclare très vaguement que ces chacinas seraient liées à des conflits entre des dealers de drogues. 
Les enfants constituent un groupe particulier, parmi les victimes des actions de " justice " illégale. Dès les années soixante-dix, leur présence dans les rues s'est accrue ; elle est devenue extrêmement visible dans les années quatre-vingt. Ce phénomène complexe a induit deux conséquences importantes : d'un côté, des réactions de violence à leur encontre, d'une très grande virulence; de l'autre, une évolution radicale des modes de prise en charge institutionnelle de cette population extrêmement vulnérable. Revenir à la partie précédente

\section{NOTES}

1. Il s'agit d'accrocher le prisonnier au " perroquet ", méthode de torture courante dans les commissariats de police brésiliens. Le " perroquet " est une barre suspendue à laquelle la personne est attachée pendant des heures, pieds et mains liés.

2. Roberto Da Matta a attiré l'attention sur la façon méprisante qui caractérise l'emploi des termes " individu " et " citoyen " dans le vocabulaire policier brésilien. Le mot " baraque " rappelle ici les habitations en bois des favelas et désigne d'une manière générale le logement typique de la population à bas revenus. Cf. Da Matta Roberto, Carnavals, bandits et héros. Ambiguïtés de la société brésilienne, Paris, Seuil, 1983.

3. . Les données citées par José Toledo proviennent du Datasus, la banque de données du Ministère de la santé, cf. Toledo José Roberto de, " Em 15 anos, homicidios triplicam no Brasil ", Folha de São Paulo, 11.11.1996. Des données de l'Organisation

Panaméricaine pour la Santé font mention d'un taux de dix-sept homicides pour cent mille habitants au Brésil en 1988, contre soixante-treize pour la Colombie en 1989, neuf pour les Etats-Unis et deux pour le Canada, en 1989 également (cf. Akerman Marco (coord.), Mapa de Risco da Violência. Cidade de São Paulo, São Paulo, CEDEC, 1996. Fernando Godinho et Rui Nogueira, à partir d'une étude diffusée par la Banque Mondiale, indiquent pour le Brésil le taux de 24,29 homicides pour cent mille habitants, cf. Godinho Fernando e Nogueira Rui, " Estudo mostra que so Colômbia é mais violenta do que o Brasil ", Folha de São Paulo, 18.3.1996.

4. Caldeira Teresa Pires do Rio, op. cit.

5. L'une des expressions majeures de ce changement est le passage d'une approche purement juridique de la criminalité à une approche sociologique du même problème, qui est devenue dominante. La réorientation du débat, enregistrée par Sergio Adorno a accompagné l'évolution du phénomène, cf. Adorno Sergio, " A Criminalidade Urbana Violenta no Brasil : Um Recorte Tematico ", Rio de Janeiro, BIB, n 35, 1er semestre 1993, p. 3.

6. pour un bilan de cette littérature, voir Sergio Adorno, op. cit.

7. Cette expression renvoie à un phénomène relativement courant, surtout à Rio de Janeiro, les décès causés par des balles tirées de façon aléatoire, et qui ne visaient pas nécessairement la personne touchée. Cf. Lozano André et Schlegel Rogerio, "

Assassinato apavora 63 \% das pessoas ", dans Folha de São Paulo, 23.6.1996.

8. L'auteur fait référence à une étude développée au Centre d'Etudes de la Violence de l'Université de São Paulo (NEV-USP) et coordonnée par Guaracy Mingardi. Cf. Ibid. 
9. 93,9\% des assassins et 90,6 \% des victimes, dans l'étude sur la zone sud de São Paulo, coordonnée par Mingardi, et citée par André Lozano, cf. André Lozano, Schlegel Rogerio, op. cit.

10. Caldeira Cesar, " Operaçao Rio e Cidadania : as Tensoes entre o Combate à Criminalidade e a Ordem Juridica ", Politica e Cultura. Visoes do Passado e Perspectivas Contemporâneas, São Paulo, Hucitec/ANPOCS, 1996, p. 56.

11. Barcellos Caco, Rota 66. A historia da policia que mata, São Paulo, Editora Globo, 1992

12. Toledo José Roberto de, " Em 15 anos, homicidios triplicam no Brasil ", Folha de São Paulo, 11.11.1996.

13. Benevides Maria Victoria, "Violência, povo e policia ", Violência urbana no noticiario de imprensa, São Paulo, Brasiliense 1983.

14. O Estado de São Paulo, 17.7.1980

15. Benevides Maria Victoria, ibid, p. 38.

16. Ibid, p. 84.

17. O Globo, le 2.12.1977.

INDEX

Mots-clés : violence, villes/ sociologie urbaine, démocratie Index géographique : Amérique du Sud, Brésil 